\title{
Artefacts Removal From Optical Coherence Tomography Angiography
}

\author{
Ee Ping Ong, Jun Cheng, Ying Quan, Guozhen Xu, Damon W.K. Wong \\ Institute for Infocomm Research, Agency for Science, Technology and Research, Singapore \\ \{ epong, jcheng, quanying, xug, wkwong \}@i2r.a-star.edu.sg
}

\begin{abstract}
This paper presents a new approach for artefacts removal from optical coherence tomography angiography (OCTA). The artefacts mainly arise as a result of distortion due to eye movements during OCT scanning process. These distortions manifest themselves as visible motion artefacts when doctors review the enface image of OCTA data. To remove these artefacts, firstly we perform motion registration for the captured OCT volume data and subsequently perform motion correction to obtain the registered OCT data. Next, we compute the OCTA from the registered OCT data using an enhanced correlation mapping technique. Thereafter, we compute the enface image from the OCTA data. In the next step, we attempt to locate regions where there is misalignment in the OCT frames of the various B-scans. Finally, we attempt to restore the regions where correct data is postulated to be absent. Our experimental results demonstrate the effectiveness of our proposed approach.
\end{abstract}

\section{Introduction}

Optical coherence tomography (OCT) has become a commonly used imaging modality in ophthalmology that non-invasively generates images of the retina in cross section or in three dimensions. Advances in OCT technology have facilitated additional applications of OCT such as optical coherence tomography angiography (OCTA), which is a new non-invasive imaging technique to generate $3 \mathrm{D}$ angiographic images from optical coherence tomography volumetric images (that takes only a matter of seconds to generate). Such technique detects motion of blood flow as motion contrast from several sequential OCT scans taken at the same cross-section to construct a map of blood flow. OCTA methods to image blood flow may be achieved by measuring phase and/or intensity variations of the OCT volumetric images with multiple repeated OCT scans. As a result, OCT angiography is non-invasive and does not require the intravenous injection of contrast dyes like other angiography imaging techniques such as Fluorescein angiography (FA) and indocyanine green angiography (ICGA) where imaging takes up to 10-30 minutes. The administration of dyes is time consuming, and can also cause adverse side effects such as nausea and vomiting, in up to 25 percent of patients (and rarely also anaphylaxis), which makes it impractical to be used as a standard clinical tool. Consequently, OCTA provides significant ad-

X. Chen, M. K. Garvin, J. Liu, E. Trucco, Y. Xu (Eds.): OMIA 2016, Held in Conjunction with MICCAI 2016, Athens, Greece, Iowa Research Online, pp. 73-80, 2016. Available from: http://ir.uiowa.edu/omia/2016_Proceedings/2016/ 
vantages compared to FA and ICGA and is a promising imaging modality for noninvasive evaluation of common ophthalmologic diseases $[1,11]$.

In general, because the 3D OCT volume data takes a few seconds to acquire, there will usually be distortion due to eye movements during the OCT scanning process. These distortions will manifest themselves as visible motion artefacts when doctors review the OCT volume data (usually in the form of enface image). As a result, methods have been proposed to remove these distortions. For example, in [8], the authors proposed to perform image registration on a per A-scan basis using orthogonal scan patterns and then perform motion correction using the image registration information in an attempt to reduce or even eliminate these motion distortions.

In [12], the authors provided a review and comparison of various algorithms for optical coherence tomography angiography, including split-spectrum amplitudedecorrelation angiography, correlation matching OCT, optical microangiography, speckle variance, and phase variance methods. In [6], the authors postulated that optic nerve head $(\mathrm{ONH})$ blood flow may be associated with glaucoma development and proposed the use of Split-spectrum amplitude-decorrelation angiography (SSADA) method [7] together with flow index and vessel density for glaucoma detection. The split-spectrum amplitude-decorrelation angiography method [7] detects motion of blood flow as decorrelation of signals' amplitude between multiple sequential OCT B-scans taken at the same cross-section to construct a map of blood flow. In this approach, the full OCT spectrum was split into several narrower bands. Inter-Bscan decorrelation was computed using the spectral bands separately and then averaged.

The correlation matching OCT (cmOCT) method [3,9] allows differentiation of flow regions since the static regions will have high correlation values while the flow regions will usually have low correlation values. The cmOCT method is achieved solely through the processing of the OCT amplitude and does not require phase information. To compute the OCT angiography, firstly, two full-range amplitude OCT images were calculated by averaging the two consecutive frames of the four OCT images captured at the same lateral position. Then, the correlation between these two full-range OCT B-frames is determined by cross-correlating a grid from frame A (average of frames 1 and 2) to the same grid position from frame B (average of frames 3 and 4) [9].

\section{Proposed Method}

Our proposed scheme operates as follow:

1. Perform motion registration of the OCT data followed by motion correction.

2. Compute the optical coherence tomography angiography (OCTA).

3. Compute the OCTA enface image and locate regions where there is misalignment in the OCT B-scans due to motion distortion, and then restore these regions.

The details of our proposed approach are as described in the following sections. 


\subsection{Motion Registration and Motion Correction}

In our proposed scheme, in the first step we first perform registration of the OCT volumetric data. More specifically, we perform the image registration or alignment between two adjacent B-scan slices by a global alignment, where a translation $(\Delta x, \Delta y)$ including both horizontal and vertical directions is applied on the entire slice.

Let $I_{i}$ be the reference slice, and $I_{i+1}$ be the adjacent slice to be registered/aligned, where $i=1,2, \ldots, m$, we try to find the alignment $\left(\Delta x_{i}, \Delta y_{i}\right)$ between $I_{i}$ and $I_{i+1}$ such that their differences is minimized as follows:

$$
\min _{\left(\Delta x_{i}, \Delta y_{i}\right)}=\sum_{(x, y)}\left|I_{i}\left(x+\Delta x_{i}, y+\Delta y_{i}\right)-I_{i+1}(x, y)\right|
$$

There are numerous methods to perform matching and registration. Considering that a full search will give better results but is too time consuming, we used the iterative diamond search matching strategy [14] because of its fast computation and easy implementation. In this diamond search matching method, the algorithm iteratively searches among the neighboring points for the alignment between current and reference slice until the error in between is no longer decreasing. After estimating the alignment vectors for best match/registration, we compute the registered OCT slices by performing motion correction using the estimated motion vectors to remove motion misalignment between these slices.

\subsection{Optical Coherence Tomography Angiography Computation}

In our second step, we compute the optical coherence tomography angiography from the registered OCT data using an enhanced correlation mapping technique. Firstly, we compute the correlation for consecutive frames of B-scans [9] using the following equation (2):

$$
C(x, y, z)=\sum_{p=0}^{M} \sum_{q=0}^{N}\left(\frac{\left[I_{A}(x+p, y+q, z)-\overline{I_{A}(x, y, z)}\right]}{\sqrt{\left[I_{A}(x+p, y+q, z)-\overline{I_{A}(x, y, z)}\right]^{2}\left[I_{B}(x+p, y+q, z)-\overline{\left.I_{B}(x, y, z)\right]^{2}}\right.}}\right)
$$

where $M$ and $N$ are the grid size, $I_{A}$ and $I_{B}$ are the two B-frames at the same location, and $\bar{I}$ is the grid's mean value. This grid is then shifted across the entire XY OCT image and a two-dimensional correlation map is generated. The resulting correlation map obtained contains values in the range of -1 to +1 which indicates weak correlation and strong correlation respectively. Then, we take the average of all the correlation frames, i.e. the correlation measures between frame 1 and frame 2, frame 2 and frame 3, and frame 3 and frame 4 . This is different and in contrast to the method proposed in [9] where correlation is computed between the two OCT B-frames obtained by the averaging of frames 1 and 2 and the averaging of frames 3 and 4 . We found that our proposed approach produces better results.

\subsection{Locate Regions of Motion Distortion and Removal of Motion Artefacts}

In our third step, we first compute the enface image $E$ from the OCTA using the following equation: 


$$
E(x, y)=\sum_{z=1}^{Z} C(x, y, z)
$$

where $\mathrm{Z}$ is the number of pixels in each A-scan. Enface OCT is one of the OCT visualization approaches [5] and an enface OCTA image appears similar to those captured using other angiography imaging techniques such as Fluorescein angiography (FA) and indocyanine green angiography (ICGA). Enface OCT image was reported to offer the benefits and ability to more easily diagnose and monitor eye disease [5]. Note that after computing the OCTA enface image, we will usually observe dark horizontal strips in this enface image. These regions appear as a result of existing misalignment between the B-frames of the OCT B-scans which cannot be effectively removed from motion correction.

Next, we attempt to locate regions where there is misalignment in the OCT frames of the various B-scans due to motion distortion. Such misaligned regions will characteristically appear as dark horizontal strips in the OCTA enface image. We achieve this by first computing the mean of the correlation enface image $E$ in the horizontal direction, denoted as:

$$
v(y)=\frac{1}{M} \sum_{x=1}^{M}(E(x, y))
$$

Then, we compute the threshold $T$ as:

$$
T=\left[\frac{1}{N} \sum_{y=1}^{N}(v(y))+\min _{y}(v(y))\right] / 2
$$

The values of $v$ which is less than the threshold $T$ can be regarded as regions with existing misalignment that existed even after motion correction. We henceforth created a mask with white pixels at these regions where $v$ is less than $T$. More specifically, what we do is that we assign a line of horizontal white pixels at rows with $v$ less than $T$ in the mask.

Finally, we attempt to restore these regions where motion distortions are present. Here, we adopted the method of [2] together with the mask we have created in the previous step to perform region filling of the masking regions (these are the regions where there is misalignment in the OCT frames of the various B-scans and where motion registration failed to register the various B-scans, resulting in horizontal dark lines/strips when we view the enface image visually). This region-filling method is an exemplar-based texture cum structure synthesis approach where we try to fill in the masking regions with texture that is similar to the surrounding regions and yet taking into consideration the linear structures (such as edges) present in the vicinity.

\subsection{Objective Similarity Measurements}

To validate the effectiveness of our proposed approach, some form of objective quality or similarity measurements with respect to the ground truth data is necessary. There are many different ways of computing similarity and we have adopted 2 commonly used metrics, namely: peak signal to noise ratio (PSNR) [4] and structure similarity index (SSIM) [13]. For PSNR, the higher the value is, the closer the similarity 
or match. On the other hand, the SSIM has a value between -1.0 to 1.0 , and the closer the value is to 1 indicates the better the match.

PSNR (or its functional inverse, the mean square error (MSE)) is the most traditionally used similarity metric. Because of this, we have included this metric in our study for general reference. On the other hand, SSIM is a relatively newer metric that has become popular in recent years due to its well-recognized good performance through the validation of various independent researchers. As a result, SSIM has also been adopted by Video Clarity into their product "ClearView" [10] (this is a commercially available product for video quality measurement and analysis).

\section{Results}

Our proposed approach has been tested on 3D OCT volumetric data captured using TopCon DRI OCT-1 Atlantis machine. This is a commercially available swept-source OCT machine with $1,050 \mathrm{~nm}$ wavelength and scanning speed of 100,000 A-scans/sec. This OCT machine performs A-scans of the OCT volume in the axial direction, and transverses in the $x$-direction to perform a B-scan. The machine will capture 4 frames of each B-scan at the same location. We have captured many 3D OCT volume data from numerous subjects using the afore-mentioned TopCon DRI OCT-1 Atlantis machine. Our analysis of the captured OCT volume data reveals that apparently motion artefacts are always present to some extend in the captured OCT volume data. The presence of the motion artefacts can be more readily observed if we scrutinize the enface image of the OCT volume data (instead of the raw 3D OCT data).

Fig. 1 shows an example of an enface image of a 3D OCT volume data captured from a subject (mainly focused on the optic disc). The motion artefacts that manifested as misalignment of the retina blood vessels can be observed in this enface image. Fig. 2 shows the enface image of a 3D OCTA volume computed using the correlation mapping technique. It can be observed that the OCTA looks similar to FA/ICGA but without the need for intravenous injection of contrast dyes. We can see that the OCTA is able to highlight retina blood vessels which cannot be observed in the enface image of the OCT volume data.

In an attempt to remove or at least reduce the motion artefacts, we performed motion registration on the 3D OCT volume data followed by motion correction. Fig. 3 shows an enface image of the registered 3D OCT volume while Fig. 4 shows the enface image of the registered 3D OCTA volume. It can be seen that some of the very obvious misalignment of the retina blood vessels have been reduced significantly. However, some obvious dark horizontal strips which are due to motion artefacts (as a result of motion distortion) are visible in this OCTA enface image.

Using our proposed technique, our algorithm is able to automatically identify the regions where motion distortions have occurred and create a mask marked with white pixels to represent these regions. Then, we attempt to restore these regions where motion distortions are present by using the method proposed in [2] to perform region filling of the masking regions. The final result, being the enface image of the registered OCTA volume with the artefacts removed, is shown in Fig. 5(a), where it can be 
seen that the dark horizontal strips corresponding to the regions with motion distortions have been effectively eliminated (to a certain extent).

For the sake of comparison, we show in Fig. 5(b) the corresponding part of the colour fundus image that corresponds to the OCTA enface image. It can be seen that the OCTA enface image is able to show the smaller retina blood vessels that is not visible in the colour fundus image.

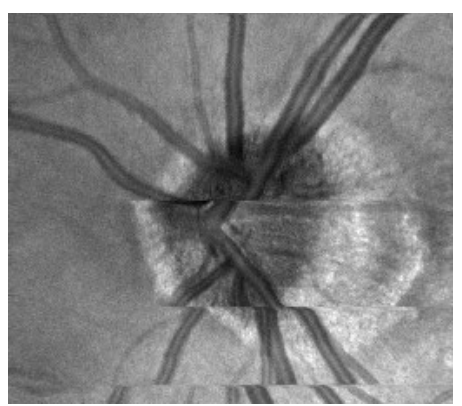

Fig. 1. Enface image of OCT volume.

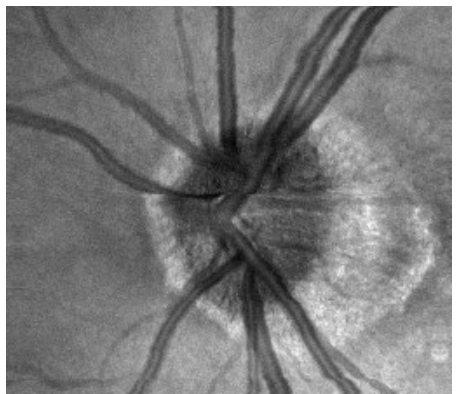

Fig. 3. Enface of registered OCT volume.

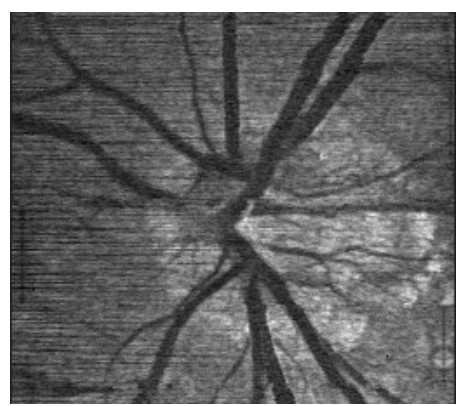

(a)

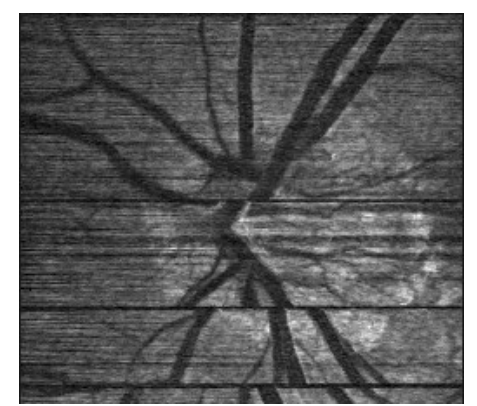

Fig. 2. Enface image of OCTA volume.

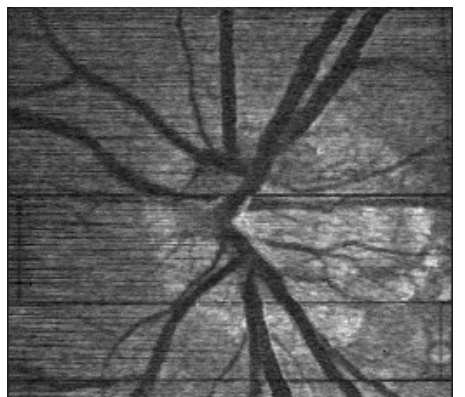

Fig. 4. Enface of registered OCTA volume.

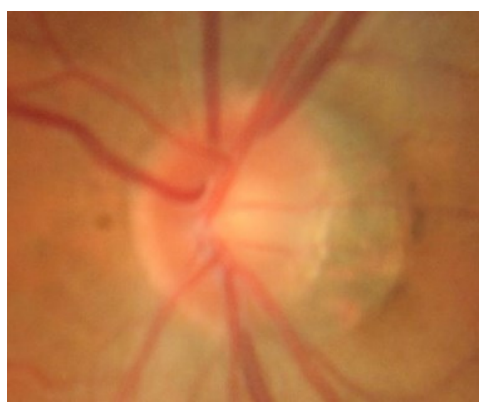

(b)

Fig. 5. (a) Enface image of registered OCTA volume with the artefacts removed; (b) Corresponding part of color fundus image.

One of the main issues we faced with validating our work is how to obtain the ground truth data so that we will be able to make objective comparison with. As there 
is no ground truth data readily available, so in our limited capacity we attempted to generate ground truth data on our own by first capturing multiple OCT 3D volumes for each eye of the subject (we used 3 OCT capture for each eye in our experiments). Thereafter, we performed supervised registration of all the OCT 3D volumes to obtain an approximate alignment of all the B-scans. Next, we used these OCT data to compute the OCTA and finally generate an enface image of the registered and aligned OCTA. We then regard this OCTA enface image as the ground truth data. As the supervised registration of multiple captures of OCT 3D volumes for an eye are tedious and time consuming, we only performed this task for 10 eyes and hence obtained 10 sets of OCTA enface images as the ground truth data.

We also captured one more OCT 3D volume for each eye of the same subject and used these to compute the non-registered OCTA volume, the registered OCTA volume (obtained using our proposed method) and also the registered OCTA volume with the artefacts removed (after applying our artefacts removal scheme). Thereafter, we compute the enface image for each case and then compute the similarity scores with the respective ground truth OCTA enface image. The average scores of the objective similarity measurements (comprising of PSNR and SSIM scores) are tabulated as shown in Table 1.

Table 1. Objective similarity scores with respect to ground truth data.

\begin{tabular}{|l|c|c|}
\hline Method & PSNR (dB) & SSIM \\
\hline Enface of OCTA volume (not registered) & 17.44 & 0.916 \\
\hline Enface of registered OCTA volume & 18.12 & 0.930 \\
\hline Enface of registered OCTA volume with artefacts removal & 18.45 & 0.938 \\
\hline
\end{tabular}

Note that when computing the similarity scores, we have discarded a small strip of pixels at the four borders of the enface image to ensure that errors introduced because of OCTA computation at the edge of the image will not be included. From Table 1, we can see that both the PSNR score and the SSIM score for the enface image of the registered OCTA volume (without artefacts removal) are higher than the nonregistered OCTA volume. In addition, we can also see that both the PSNR score and the SSIM score for the enface image of the registered OCTA volume with artefacts removal is higher than the registered OCTA volume without artefacts removal. These results show that registration of the OCTA volume using our approach make them more similar to the ground truth, while our artefacts removal approach makes the enface image of the registered OCTA volume even more similar to the ground truth OCTA enface image. These results clearly illustrate the usefulness of our proposed approach.

\section{Conclusions}

In this paper, we have presented a new approach for artefacts removal from optical coherence tomography angiography. The optical coherence tomography angiography is computed from optical coherence tomography $3 \mathrm{D}$ volume data captured using a commercial swept-source OCT machine. Here, we have shown how we are able to achieve this using motion registration and correction of captured OCT $3 \mathrm{D}$ volume 
data, and also an enhanced correlation mapping technique to obtain better optical coherence tomography angiography. We have also demonstrated our proposed method to automatically identify regions of misalignment that still existed in the OCT frames of the various B-scans (after motion correction) and finally the method we use to restore the misaligned/motion-distorted regions. Our experimental results show the effectiveness of our proposed approach.

\section{References}

1. Carlo TE, Romano A., Waheed N.K., Duker J.S.: A review of optical coherence tomography angiography (OCTA). International Journal of Retina and Vitreous 1(1) (2015)

2. Criminisi, A., Perez, P., Toyama, K.: Region filling and object removal by exemplar-based image inpainting. IEEE Trans. on Image Processing 13(9), 1200-1212 (2004)

3. Enfield, J., Jonathan, E., Leahy, M.J.: In vivo imaging of the microcirculation of the volar forearm using correlation mapping optical coherence tomography. Biomedical Optics Express 2(5), 1184-1193 (2011)

4. Eskicioglu, A., Fisher, P.: Image quality measures and their performance. IEEE Transactions on Communications, Vol. 43(12), 2959-2965 (1995)

5. Heiferman, M., Simonett J., Fawzi, A.: En Face OCT Imaging in Retinal Disorders. Retinal Physician 12, 45-48, (2015)

6. Jia Y, Morrison JC, Tokayer J, Tan O, Lombardi L, Baumann B, Lu CD, Choi W, Fujimoto JG, Huang D.: Quantitative OCT angiography of optic nerve head blood flow. Biomedical Optics Express 3(12), 3127-3137 (2012)

7. Jia Y, Tan O, Tokayer J, Potsaid B, Wang Y, Liu JJ, Kraus MF, Subhash H, Fujimoto JG, Hornegger J, Huang D.: Split-spectrum amplitude-decorrelation angiography with optical coherence tomography. Opt Express. 20(4):4710-4725 (2012)

8. Kraus, M.F., Potsaid, B., Mayer, M.A., Bock, R., Buamankn, B., Liu, J.J., Hornegger, J., Fujimoto, J.G.: Motion correction in optical coherence tomography volumes on a per Ascan basis using orthogonal scan patterns. Biomedical Optics Express 3(6), 1182-1199 (2012)

9. Subhash, H.M., Leahy, M.J.: Microcirculation imaging based on full-range high-speed spectral domain correlation mapping optical coherence tomography. Journal of Biomedical Optics 19(2), 021103-1 (2014)

10. Video Clarity.: White Paper: Advancing To Multi-Scale SSIM. http://videoclarity.com/videoqualityanalysiscasestudies/ (2012)

11. Waheed N.K., Carlo T.E., Chin A.T., Duker J.S.: OCT Angiography in retinal diagnosis and treatment. Retina Physician 12, $42-46$ (2015)

12. Zhang, A., Zhang, Q., Chen, C.L., Wang, R.K.: Methods and algorithms for optical coherence tomography-based angiography: a review and comparison. Journal of Biomedical Optics 20(10), pp. 100901-1 10091-13 (2015)

13. Zhou, W., Bovik, A.C., Sheikh, H.R., Simoncelli, E.P.: Image quality assessment from error visibility to structural similarity. IEEE Trans. on Image Processing 13(4), 600-612 (2004)

14. Zhu, S., Ma, K.: A new diamond search algorithm for fast block-matching motion estimation. IEEE Trans. on Image Processing 9, 287-290 (2000) 